\title{
Adherence to a new oral anticoagulant treatment prescription: dabigatran etexilate
}

\author{
This article was published in the following Dove Press journal: \\ Patient Preference and Adherence \\ 30 June 2009 \\ Number of times this article has been viewed
}

\section{Bellamy' \\ N Rosencher' \\ BI Eriksson ${ }^{2}$}

'Anaesthesiology Department, Hôpital Cochin (AP-HP), René

Descartes University, Paris 750I 4

France; ${ }^{2}$ Orthopaedic Department,

University Hospital Sahlgrenska/Ostra,

Gothenburg, Sweden
Correspondence: Nadia Rosencher Anaesthesiology Department, Hôpital Cochin (AP-HP), René Descartes University, Paris 75014 France Email: nadia.rosencher@cch.aphp.fr

\begin{abstract}
The recent development of new oral anticoagulants, of which dabigatran etexilate is currently at the most advanced stage of development, is the greatest advance in the provision of convenient anticoagulation therapy for many years. A new oral anticoagulation treatment, dabigatran etexilate, is already on the market in Europe. The main interest probably will be to improve the prescription and the adherence to an effective thromboprophylaxis in medical conditions such as atrial fibrillation without bleeding side effects, without the need for monitoring coagulation, and without drug and food interactions such as vitamin K anticoagulant(VKA) treatment. Dabigatran is particularly interesting for extended thromboprophylaxis after major orthopedic surgery in order to avoid daily injection for a month. However, oral long-term treatments such as VKA are not systematically associated with a higher compliance level than injected treatments such as low-molecular-weight heparins. Indeed, adherence to an oral treatment, instead of the usual daily injection in major orthopedic surgery, is complex, and based not only on the frequency of dosing but also on patient motivation, understanding, and socio-economic status. New oral anticoagulants may be useful in this way but education and detection of risk factors of nonadherence to treatment are still essential.
\end{abstract}

Keywords: oral anticoagulant, adherence, compliance, education, dabigatran

\section{Introduction}

New oral anticoagulants are being promoted, but some observers have expressed concern about adherence to these oral therapies in comparison with low-molecular-weight heparin (LMWH) injected by nurses or by patients themselves.

Nonadherence or incomplete adherence to drug prescriptions is common. In long-term therapy for chronic disease (such as hypertension and myocardial infarction), it has been estimated that only half of all drug doses are taken as prescribed. ${ }^{1,2}$ Oral anticoagulant vitamin $\mathrm{K}$ antagonist (VKA) therapy probably has the same problems with adherence, although no coagulation monitoring is necessary with dabigatran etexilate (dabigatran). However, prescription of dabigatran after orthopedic surgery does not exceed 4 weeks, and thus the adherence should be greater than for VKA, which is usually prescribed for a period of many years. The problem is the clinical significance of the impact of nonadherence. Furthermore, efficacy of any self-administered medication, particularly with injection, depends to a large extent on patient compliance.

\section{Compliance with oral treatment Anticoagulation treatment compliance}

Long-term anticoagulation mainly concerns medical issues such as prevention of stroke or venous thromboembolism event (VTE) treatment. In these cases VKA is used. 
During these treatments coagulation needs to be monitored frequently so that the dose can be adapted to overcome the problem of patients receiving chronic VKA therapy who have poor anticoagulation control and an increased risk of adverse events. Adherence, but also medications and food interactions, a small therapeutic window, and many other variables lead to the need for frequent anticoagulation monitoring with VKA. According to the pharmacokinetic profile, the need for monitoring coagulation is higher and adaptation is more difficult when half-life of VKA is short. Because warfarin has a longer half-life than acenocomarol, warfarin is much more often used. ${ }^{3}$ Despite good compliance with VKA (60\%), anticoagulation control is often inadequate, leading to a risk of bleeding complications. ${ }^{4-6}$

Daily LWMH injections are more often used after major surgery such as orthopedic surgery. Daily self injection of LWMH increases the risk of nonadherence, but this risk has not been properly evaluated. Furthermore, platelets monitoring is required in France twice a week during the first month and once a week thereafter. Adherence to platelets monitoring has also not been evaluated.

\section{Compliance with others drugs}

As medicines become more effective, access to healthcare and patient noncompliance will become the leading causes of treatment failure. Haynes et $\mathrm{al}^{7}$ define patient compliance as “the extent to which a person's behaviour, in terms of taking medications, following diets, or executing lifestyle changes, coincides with medical or health advice."

Most patient noncompliance factors are common to all patients with chronic conditions, including patients treated with VKA.

Many factors associated with poor compliance have been identified in several studies: frequent dosing, patient perception of treatment benefits, poor patient-physician communication, lack of motivation, poor socioeconomic background, lack of family and social support, young age, and predementia patients recently diagnosed as having a chronic illness. ${ }^{8}$ Furthermore, a more specific study about warfarin long-term treatment ${ }^{1}$ has shown that extra-medical parameters (such as marital status, living arrangements, social problem) interfere with VKA medication (and other medications) and lead to noncompliance.

Several studies have found an inverse relationship between the number of doses prescribed and compliance. Compliance declines as dosage frequency increases. ${ }^{9-11}$ In a study of diabetic patients taking oral hypoglycemic agents, compliance rates were $74.8 \%$ for once-daily doses and $38 \%$ for thrice-daily doses. ${ }^{12}$ On the other hand, the severity of disease or the gravity of outcome does not improve treatment compliance. In patients with chronic disease, compliance rates tend to decrease over time.

\section{Impact of nonadherence}

Poor compliance is probably the most common cause of failure to respond to medications and poor treatment outcomes. ${ }^{13}$ In a clinical trial, noncompliance has been reported to have little impact on treatment efficacy, leading to false conclusions about the efficacy of a potentially useful drug. ${ }^{14}$ But poor compliance often leads to additional tests, changes in the treatment plan, emergency treatment, or hospitalization, ${ }^{15}$ which increase the cost of medical care.

\section{Dabigatran etexilate adherence}

It is interesting to note that between 1 and 4 hours after the surgery, the patient is not always able to swallow any medication because of nausea. In this case, we recommend starting with an injection of LMWH on the first day to avoid any risk of nonefficacy.

For dabigatran, one of the nonadherence risks is a result of two capsules needing to be taken together once a day. Another risk is the possibility of skipping a dose or taking two different doses on the same day. Skipping one dose is less important, because the rate of deep vein thrombosis (DVT) after discharge decreases, but the risk of a double dose is much more dangerous, and only good information at the time of prescribing can resolve or decrease this risk. In comparison with VKA, dabigatran probably has a lower risk because of its wider therapeutic window due to the specificity (antithrombin exclusive) of this drug.

After asking patients about preference for oral or subcutaneous injection, we have to inform and convince them about the risk of skipping or taking a double daily dose of an oral anticoagulant. Moreover, physicians should suggest ways of improving home adherence such as the use of a pillbox, commonly used in confused or elderly patients.

Therefore, with good communication and education, especially for patients at risk of nonadherence to a drug such dabigatran, dabigatran could be at least as effective and safe as VKA.

All authors agree on the benefits of improving rehabilitation, by whatever means. Indeed, considering current knowledge of surgical physiopathology, the primary issues in the early postoperative phase pertain to analgesia, mobilization, acute respiratory and cardiovascular issues, delirium, antiemetic treatment and nutrition. ${ }^{16}$ For instance, if pain relief is 
controlled the risk of death is significantly reduced in patients given combined general anesthesia and peripheral nerve block compared with those who receive only general anesthesia or only spinal anesthesia. The beneficial effect of the combined use of general peripheral nerve block may be because this approach reduces the quantity of general analgesic used to control pain and reported pain levels, enabling earlier functional recovery. ${ }^{16-22}$ It has been demonstrated that improving rehabilitation can decrease mortality by $50 \% .{ }^{17}$ Therefore, the lack of an injection should also improve rehabilitation for many physical and psychological reasons: faster recovery of autonomy, and feeling recovered without injection and no dependence on a nurse for daily injection.

\section{How to improve adherence}

One of the easiest ways to improve adherence is probably patient education, although in a recent small, effective, randomized study, Laporte et $\mathrm{al}^{3}$ failed to prove a statistical relationship between education and compliance. A meta-analysis of educational programs in adult asthmatics confirmed that such programs are associated with improved compliance..$^{23}$

The constant demand on physicians for more rapid patient turnover has significantly reduced the time spent with each patient. Therefore, the use of nurses and para-medical staff to assist patient education and follow-up has been advocated. More studies are needed to assess the efficacy of educational programs by nurse educators. Special attention should be paid to persons receiving three or more drugs, living alone, receiving drugs from other doctors, and to persons with predementia symptoms, as they are at higher risk of nonadherence. Physicians should establish a partnership with the patient and their family in these cases. Other ways of improving adherence are reducing the number and frequency of pills prescribed, developing individualized treatment plans, ${ }^{24}$ and helping patients develop ways of remembering to take each dose. Calendar blister packaging seems to improve medication compliance, particularly in the elderly and in those with a history of noncompliance. ${ }^{25}$ Telephone-based management of oral anticoagulation through a pharmacist-staffed anticoagulation clinic yielded clinical outcomes that were at least as favorable as those associated with traditional office-based visits. ${ }^{26}$ In general, a combination of different strategies works better than a single strategy, and long-term interventions may be necessary to increase compliance.

\section{The risk}

As noted previously, the risk of forgetting one dose of anticoagulant in the postoperative period is not very high after discharge because of the decreasing risk of VTE. But the risk of forgetting beta-blockers or hypotension medication is much higher in elderly patients. Indeed, the risk of myocardial infarction or stroke is obviously higher if treatment is skipped. Many countries adopt the practice of stopping anticoagulation treatment at discharge or after 7 days, despite all recommendations, probably because of painful injection related to taking of LMWH, and thus convenience is very important. Furthermore, prescribing aspirin to prevent DVT after discharge is not unusual, even if the effect is known to be inadequate in these indications and all recommendations warn against prescribing aspirin for DVT prophylaxis.

If a double dose of hypotension medication is given, the risk of severe hypotension can be more dangerous than a major bleeding after taking an anticoagulant. Because elderly or confused patients often take many medications, they usually use a pill box. Dabigatran or any other treatment can be inserted into this pill box with other medications. The risk of bleeding is related especially to age and renal function. Therefore, ACCP guidelines recommend ${ }^{27}$ reducing the dose of anticoagulant in this population. But there are no recommendations on the percentage dose reduction necessary, because studies on lower-dose LMWH have been inadequate to demonstrate efficacy. However, the European Medicines Agency (EMEA) recommends a low dabigatran dose of $150 \mathrm{mg} /$ day in elderly patients, or those with significant renal impairment $\left(\mathrm{CL}_{\mathrm{CR}} 30\right.$ to $\left.50 \mathrm{~mL} / \mathrm{min}\right)$, or amiodarone treatment. Indeed, this lower dose has been validated by studies. ${ }^{28,29}$

\section{Dabigatran}

Dabigatran, a novel, oral, reversible direct thrombin inhibitor, is being investigated for several thromboembolic diseases and was approved by the EMEA in March 2008 for the prevention of VTE in adult patients undergoing elective total hip or knee replacement. The onset and offset of its anticoagulant activity are rapid and predictable. In the clinical phase III program, dabigatran (started between 1 and 4 hours after surgery, once a day) was found to be as effective as enoxaparin (40 mg once a day, started 12 hours prior to surgery for the primary prevention of VTE), and with a similar safety profile, in the two phase III trials RE-MODEL ${ }^{30}$ in Total Knee Replacement (TKR) and RE-NOVATE ${ }^{31}$ in Total Hip Replacement which led to approval in the European Union. Dabigatran is an oral alternative to subcutaneous enoxaparin for the prevention of VTE after total knee and hip replacement. At this time, pivotal studies for VTE treatment and prevention of stroke 


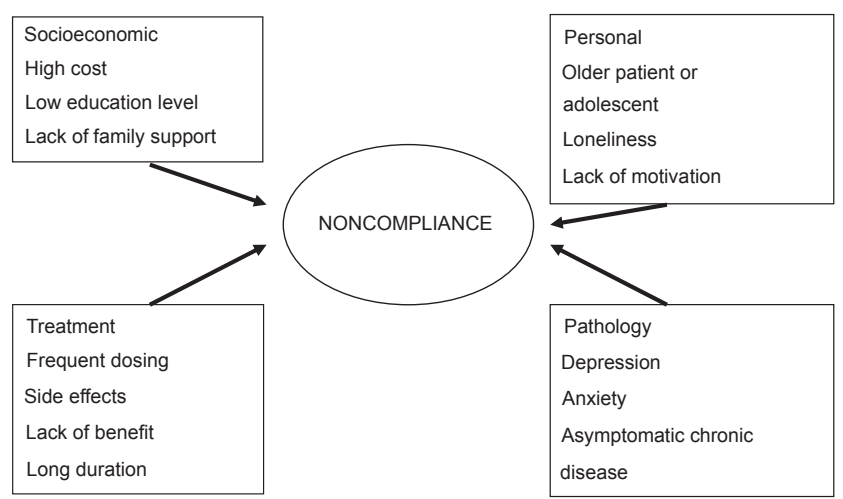

Figure I Patient noncompliance risk factors.

in patients with atrial fibrillation are completed, but have not yet been presented. This new oral anticoagulant has some major advantages over traditional anticoagulants, including a lack of need for anticoagulant monitoring and a low drug-drug interaction potential, and can be used in both the acute and chronic settings. A UK National Health Service (NHS) report indicates that only about half the patients undergoing major orthopedic surgery who are at high risk of thromboembolism complications receive effective thromboprophylaxis. Even if aspirin is not recommended ${ }^{27}$ it is often used in many countries, because it can be taken orally. The approval of dabigatran has the potential to greatly improve this situation, as it has several advantages over current treatments: it is an oral drug that can be easily administered in hospital and after discharge, and offers the prospect of a longer duration of prophylaxis with high adherence. A substudy analysis of BISTRO-II data showed that early treatment initiation (within 2 to 4 hours post surgery) was more efficacious, without increased bleeding, than delayed administration ( $>4$ hours). ${ }^{32}$ The recommended time for initiating dabigatran treatment, based on its pharmacokinetic profile, is within 1 to 4 hours post surgery, with only half a dose the day of surgery. Absorption is slow after the first postoperative dose (6 hours), probably due to alterations in gastric motility after surgery. In view of the increased bleeding risk immediately after surgery, this slow and steady absorption profile in the early postoperative period might represent an advantageous strategy by reducing the risk of postsurgical bleeding. ${ }^{33}$ Peak concentration $\left(\mathrm{C}_{\max }\right)$ occurs at about 6 hours after the first dose, which means about 7 to 10 hours after surgery. In the steady-state, absorption is more rapid and $\mathrm{C}_{\max }$ occurs about 2 hours after administration. Importantly, there was no evidence of the rebound phenomenon, which describes a hypercoagulable state that occurs after discontinuation of anticoagulant treatment.
In RE-MODEL, the incidence of acute coronary syndrome events remained low and similar between groups for the duration of the 3-month follow-up period; this observation, together with the lack of any significant between-group differences in pulmonary embolism or death during follow-up, suggest that there is no rebound effect on coagulation after completion of treatment.

Because half-life is 17 hours in elderly patients, if the patient forgets or skips one day, the danger after discharge is low. Indeed, the risk is very high during the first 7 days and decreases thereafter, especially in total knee replacement.

Dabigatran can be given once daily without dose adjustments. A linear correlation exists between prothrombin time, thrombin time, ecarin clotting time and plasma dabigatran concentration, confirming the predictability of the pharmacokinetics and pharmacodynamics of dabigatran. In the elderly population ( $>75$ years old) and in patients with significant renal impairment $\left(\mathrm{CL}_{\mathrm{CR}} 30\right.$ to $\left.50 \mathrm{~mL} / \mathrm{min}\right)$ a reduced dose of $150 \mathrm{mg}$ is recommended by the EMEA, This new approval represents an important step for increasing safety in this fragile population, with high risk of bleeding.

A dose of $220 \mathrm{mg}$ once a day is approved for other patients, including obese patients, regardless of ethnicity.

\section{Conclusion}

Once-daily treatment with the new oral, synthetic anticoagulant dabigatran, which requires no coagulation monitoring tests, shows promise for improving adherence to extended prophylaxis, to replace aspirin in some countries and LMWH in other countries. Adherence, after good patient education, should be as good as that for VKA or self-injected LWMH. Nevertheless, patients and their family must be made aware of the risks of treatment, even though the risk of DVT after one missed dose in the postoperative period is very low. The use of pill boxes is quite appropriate in some populations who are taking multiple medications.

\section{Disclosures}

Dr Rosencher: Membership on Board of Directors or advisory committees, research funding - Boehringer Ingelheim, GSK, Sanofi-Aventis, BMS, Bayer; Dr Eriksson: consultancy - Boehringer Ingelheim, Bayer.

\section{References}

1. Orensky IA, Holdford DA. Predictors of noncompliance with warfarin therapy in an outpatient anticoagulation clinic. Pharmacotherapy. 2005;25(12):1801-18018.

2. Loghman-Adham M. Medication noncompliance in patients with chronic disease: issues in dialysis and renal transplantation. Am J Manag Care. 2003;9(2):155-171. 
3. Laporte S, Quenet S, Buchmuller-Cordier A, Reynaud J, Tardy-Poncet B, Thirion C, et al. Compliance and stability of INR of two oral anticoagulants with different half-lives: a randomised trial. Thromb Haemost. 2003;89(3):458-467.

4. Scoditti U, Buccino GP, Pini M, Pattacini C, Mancia D. Risk of acute cerebrovascular events related to low oestrogen oral contraceptive treatment. Ital J Neurol Sci. 1998;19(1):15-19.

5. Barcellona D, Vannini ML, Fenu L, Balestrieri C, Marongiu F. Warfarin or acenocoumarol: which is better in the management of oral anticoagulants? Thromb Haemost. 1998;80(6):899-902.

6. Van der Meer FJ, Briet E, Vandenbroucke JP, Sramek DI, Versluijs MH, Rosendaal FR. The role of compliance as a cause of instability in oral anticoagulant therapy. Br J Haematol. 1997;98(4): 893-900.

7. Haynes RB, Taylor DW, Sackett DL. Compliance in Health Care. Baltimore: Johns Hopkins University Press. 1979:145-156.

8. Arnsten JH, Gelfand JM, Singer DE. Determinants of compliance with anticoagulation: A case-control study. Am J Med. 1997;103(1):11-17.

9. Cramer JA, Mattson RH, Prevey ML, Scheyer RD, Ouellette VL. How often is medication taken as prescribed? A novel assessment technique. JAMA. 1989;261(22):3273-3277.

10. Pullar T, Birtwell AJ, Wiles PG, Hay A, Feely MP. Use of a pharmacologic indicator to compare compliance with tablets prescribed to be taken once, twice, or three times daily. Clin Pharmacol Ther. 1988;44(5):540-545

11. Lorentz A, Eckardt K, Osswald P, Kruse C. Perioperative plasma erythropoietin levels in hip arthroplasty. Ann Hematol. 1994;68(3): 117-124.

12. Paes AH, Bakker A, Soe-Agnie CJ. Impact of dosage frequency on patient compliance. Diabetes Care. 1997;20(10):1512-1517.

13. Horowitz RI, SM. H. Adherence to treatment and health outcomes. Arch Intern Med. 1993;153:1863-1868.

14. Cramer JA. Feedback on medication dosing enhances patient compliance. Chest. 1993;104(2):333-334.

15. Tebbi CK. Treatment compliance in childhood and adolescence Cancer. 199315;71(10 Suppl):3441-3449.

16. Moore SB, Swenke PK, Foss ML, Rand JA, Cabanela ME, Kavanagh B, et al. Simplified enrollment for autologous transfusion: automatic referral of presurgical patients for assessment for autologous blood collections. Mayo Clin Proc. 1992;67(4):323-327.

17. Rosencher N, Vielpeau C, Emmerich J, Fagnani F, Samama CM. Venous thromboembolism and mortality after hip fracturesurgery: the escorte study. J Thromb Haemost. 2005;3:2006-2014.

18. Rosencher N, Vielpeau C, Emmerich J, Fagnani F, Samama CM. Venous thromboembolism and mortality after hip fracture surgery: the ESCORTE study. J Thromb Haemost. 2005;3(9):2006-2014.

19. Kehlet H. Multimodal approach to control postoperative pathophysiology and rehabilitation. Br J Anaesth. 1997;78:606-617.

20. Foss NB, Kristensen MT, Kehlet H. Prediction of postoperative morbidity, mortality and rehabilitation in hip fracture patients: the cumulated ambulation score. Clin Rehabil. 2006;20(8):701-708.
21. Lieberman D, Friger M, Lieberman D. Inpatient rehabilitation outcome after hip fracture surgery in elderly patients: a prospective cohort study of 946 patients. Arch Phys Med Rehabil. 2006;87(2):167-171.

22. Stenvall M, Olofsson B, Nyberg L, Lundstrom M, Gustafson Y. Improved performance in activities of daily living and mobility after a multidisciplinary postoperative rehabilitation in older people with femoral neck fracture: a randomized controlled trial with 1-year follow-up. J Rehabil Med. 2007;39(3):232-238.

23. Devine EC. Meta-analysis of the effects of psychoeducational care in adults with asthma. Res Nurs Health. 1996;19(5):367-376.

24. Bond WS, Hussar DA. Detection methods and strategies for improving medication compliance. Am J Hosp Pharm. 1991;48(9):1978-1988.

25. Wong BS, Norman DC. Evaluation of a novel medication aid, the calendar blister-pak, and its effect on drug compliance in a geriatric outpatient clinic. J Am Geriatr Soc. 1987;35(1):21-26.

26. Wittkowsky AK, Nutescu EA, Blackburn J, Mullins J, Hardman J, Mitchell J, et al. Outcomes of oral anticoagulant therapy managed by telephone vs in-office visits in an anticoagulation clinic setting. Chest. 2006;130(5):1385-1389.

27. Geerts WH, Bergqvist D, Pineo GF, Heit JA, Samama CM, Lassen MR, et al. Prevention of venous thromboembolism: American College of Chest Physicians Evidence-Based Clinical Practice Guidelines (8th Edition). Chest. 2008;133(6 Suppl):381S-453S.

28. Dahl O, Kurth AA, Rosencher N, Schnee J, Clements DH, Noack H, et al. $150 \mathrm{mg}$ Dabigatran etexilate once daily has a good safety profile and comparable efficacy to enoxaparin for primary prevention of venous thromboembolism after total knee or hip replacement surgery in patients older than 75 years or with reduced renal function. Blood. 2008;(supp).

29. Dahl O, Kurth AA, Rosencher N, Schnee J, Clemens A, Noack H, et al. Efficacy and safety profile of dabigatran etexilate compared with enoxaparin for the prevention of venous thromboembolism in moderately renally impaired patients after total knee or hip replacement surgery. Blood. 2008;112(ASH Annual Meeting Abstracts): Abstract 981.

30. Eriksson BI, Dahl O, Dijk V, CN, Frostick SP, Kurth, et al. A new oral anticoagulant, dabigatran etexilate, is effective and safe in preventing venous thromboembolism after total knee replacement surgery. (The RE-MODEL trial). Blood. 2006;108(11):573.

31. Eriksson BI, Dahl OE, Rosencher N, Kurth AA, van Dijk CN, Frostick SP, et al. Dabigatran etexilate versus enoxaparin for prevention of venous thromboembolism after total hip replacement: a randomised, double-blind, non-inferiority trial. Lancet. 2007;370(9591): 949-956.

32. Eriksson BI, Dahl OE, Buller HR, Hettiarachchi R, Rosencher N, Bravo ML, et al. A new oral direct thrombin inhibitor, dabigatran etexilate, compared with enoxaparin for prevention of thromboembolic events following total hip or knee replacement: the BISTRO II randomized trial. J Thromb Haemost. 2005;3(1):103-111.

33. Eriksson BI, Agnelli G, Cohen A, Dahl O, Mouret P, Rosencher N, et al. Significantly lower need for blood transfusions associated with post-operatively initiated subcutaneous melagatran/oral ximelagatran compared with enoxaparin. Thromb Haemost. 2004;92(2):428-430.
Patient Preference and Adherence

\section{Publish your work in this journal}

Patient Preference and Adherence is an international, peer-reviewed, open access journal that focusing on the growing importance of patient preference and adherence throughout the therapeutic continuum. Patient satisfaction, acceptability, quality of life, compliance, persistence and their role in developing new therapeutic modalities and compounds to

\section{Dovepress}

optimize clinical outcomes for existing disease states are major areas of interest. This journal has been accepted for indexing on PubMed Central. The manuscript management system is completely online and includes a very quick and fair peer-review system. Visit http://www.dovepress.com/ testimonials.php to read real quotes from published authors. 